\title{
Teor e composição química do óleo essencial de alpínia em razão da adubação e da disponibilidade de água no solo ${ }^{1}$
}

\author{
Maria Elvira de Rezende ${ }^{2}$ Janie Mendes Jasmim³, Geisa Paulino Caprini ${ }^{4}$, Elias Fernandes de Sousa ${ }^{5}$, \\ Jan Schripsema ${ }^{6}$, José Tarcísio Lima Thiébaut ${ }^{3}$
}

\begin{abstract}
RESUMO
A influência de diferentes adubos e disponibilidade de água no solo foi avaliada em relação ao teor e à composição química de óleos essenciais em folhas de alpínia. O experimento foi realizado em blocos casualizados, com três repetições, em esquema de parcelas subdivididas. As parcelas corresponderam a dois limites de disponibilidade de água no solo [LDA1 - redução de 75\% da capacidade total de retenção de água (CTA) - e LDA2 - redução de 50\% da CTA], e as subparcelas, aos adubos: esterco bovino, cama-de-galinha, torta-de-filtro, químico e o controle não-adubado. A disponibilidade de água no solo, assim como a adubação, não influenciou no teor e na composição química de óleos essenciais aos 12 meses após o plantio. Os principais constituintes químicos (teores) dos óleos essenciais em folhas de alpínia foram: $\alpha$-thujeno $(6,11 \%), \alpha$-pineno $(2,69 \%)$, sabineno $(16,69 \%), \beta$-pineno $(4,64 \%), \beta$-mirceno $(1,76 \%), 1,8$ cineol $(19,41 \%)$ e 1-terpinen-4-ol $(14,32 \%)$.
\end{abstract}

Palavras-chave: Alpinia zerumbet, Zingiberaceae, planta ornamental, nutrientes, planta medicinal.

\section{ABSTRACT \\ Effect of fertilization and soil water availability on content and chemical composition of alpinia essential oil}

This study evaluated the influence of different fertilizers and soil water availability on the content and chemical composition of essential oil from alpinia leaves. The experiment was arranged in a randomized block design in a splitplot scheme, with three replicates. The plots corresponded to two limits of soil water availability [LWD1 - reduction of $75 \%$ of the total water retention capacity (TWC) and LWD2 - reduction of 50\% of TWC] and the subplots to the fertilizers cattle manure, poultry bed, filter cake, chemical and the non-fertilized control. Soil water availability and fertilization did not affect either essential oil content or chemical composition 12 months after planting. The main chemical constituents (contents) found in the essential oil of alpinia leaves were $\alpha$ thujene $(6.11 \%), \alpha$-pinene $(2.69 \%)$, sabinene (16.69\%), $\beta$-pinene (4.64\%), $\beta$-myrcene (1.76\%), 1.8-cineole (19.41\%) and 1-terpinen-4-ol (14.32\%).

Key words: Alpinia zerumbet (Pers.) Burtt \& Smith, Zingiberaceae, ornamental plant, nutrients, medicinal plant.

\footnotetext{
Recebido para publicação em agosto de 2009 e aprovado em fevereiro de 2011

${ }^{1}$ Extraído da tese de Doutorado da primeira autora, apresentada ao Centro de Ciências e Tecnologias Agropecuárias (CCTA) da Universidade Estadual do Norte Fluminense Darcy Ribeiro (UENF). Fontes financiadoras: UENF, FAPERJ e FENORTE/TECNORTE.

${ }^{2}$ Engenheira-Agrônoma, Doutora. Embrapa Recursos Genéticos e Biotecnologia, Parque Estação Biológica, Final da Avenida W5 Norte, Asa Norte, 70770-900, Brasília, Distrito Federal, Brasil. melvira@uenf.br

3 Engenheiro-Agrônomo, Doutor. Universidade Estadual do Norte Fluminense Darcy Ribeiro, Laboratório de Fitotecnia, Setor de Horticultura, Avenida Alberto Lamego, 2000, Parque Califórnia, 28013-602, Campos dos Goytacazes, Rio de Janeiro, Brasil. janie@uenf.br

${ }^{4}$ Bióloga, Mestre. Universidade Estadual do Norte Fluminense Darcy Ribeiro, Laboratório de Engenharia Agrícola, Avenida Alberto Lamego, 2000, Parque Califórnia, 28013602, Campos dos Goytacazes, Rio de Janeiro, Brasil. melvira@uenf.br

${ }^{5}$ Engenheiro Agrícola, Doutor. Universidade Estadual do Norte Fluminense Darcy Ribeiro, Laboratório de Ciências Químicas, Avenida Alberto Lamego, 2000, Parque Califórnia, 28013-602, Campos dos Goytacazes, Rio de Janeiro, Brasil. melvira@uenf.br

${ }^{6}$ Farmacêutico, Doutor. Universidade Estadual do Norte Fluminense Darcy Ribeiro, Laboratório de Ciências Químicas, Avenida Alberto Lamego, 2000, Parque Califórnia, 28013602, Campos dos Goytacazes, Rio de Janeiro, Brasil. melvira@uenf.br
} 


\section{INTRODUÇÃO}

A alpínia [Alpinia zerumbet (Pers.) Burtt \& Smith] é uma monocotiledônea da família Zingiberaceae, mais conhecida no Brasil como colônia. A planta é aromática, herbácea, rizomatosa e perene, com ampla utilização para fins medicinais (Larsen et al., 1999; Lorenzi \& Souza, 2001; Lorenzi \& Matos, 2002; Matos, 2002). É indicada principalmente no tratamento da hipertensão e da ansiedade (Matos, 2002); porém, outras propriedades lhe são mencionadas: diurética, estimulante do peristaltismo intestinal, emenagoga, antiulcerogênica, ação expectorante, usada em afecções respiratórias, intestinais e renais, amigdalite, faringite, rouquidão, reumatismo, nevralgia e dores lombares e musculares (Côrrea, 1974; Alice et al., 1995; Panizza, 1997). Em virtude disso, a espécie tornou-se referência de várias pesquisas (Bezerra et al., 2000; Chaves, 2001; Lahlou et al., 2002).

O uso farmacológico dessa espécie é atribuído aos seus princípios ativos, dentre os quais se destacam o óleo essencial comumente extraído de folhas, os rizomas, as hastes e flores. Os componentes voláteis do óleo de $A$. zerumbet têm sido objeto de pesquisa de inúmeros estudos (Gottlieb et al., 1981; De Pooter et al., 1995; Zoghbi et al., 1999; Lahlou et al., 2003, Rezende et al., 2003). Dentre os constituintes químicos majoritários do óleo essencial estão o terpinen-4-ol, o 1,8-cineol e o $\gamma$-terpineno (De Pooter et al., 1995; Lahlou et al., 2003). Rezende et al. (2003) também verificaram maiores teores desses compostos no óleo essencial extraído em folhas secas de $A$. zerumbet, sendo encontrados 20,4 a 22,7\% de 1,8-cineol; 11,3 a $12,7 \%$ de $\gamma$-terpineno; 21,5 a 23,8\% de 1-terpinen-4-ol; e entre 0,7 a $1,5 \%$ de $\alpha$-terpinenol.

O terpinen-4-ol é considerado o principal constituinte químico do óleo essencial de A. zerumbet (Lahlou et al., 2002). Usando a ressonância magnética nuclear ( $\left.{ }^{13} \mathrm{C}-\mathrm{NMR}\right)$ como técnica de identificação complementar na análise de óleo essencial de A. zerumbet, Alencar et al. (1997) identificaram $\rho$-cimeno (33,1\%), 1,8-cineol (18,9\%), (E)terpinen-4-ol $(18,8 \%)$ e sabineno $(8,2 \%)$ como os constituintes em maior proporção.

As técnicas agronômicas de cultivo das Zingiberaceae são pouco conhecidas. Normalmente, as mudas são obtidas pela divisão de touceiras em qualquer época do ano. Para a espécie $A$. zerumbet, recomenda-se o cultivo a pleno sol, em canteiros ricos em matéria orgânica e com irrigações periódicas (Lamas, 2001; Lorenzi \& Souza, 2001; Lorenzi \& Matos, 2002). Estudando os efeitos de diversos adubos orgânicos e inorgânicos em A. zerumbet, Rezende et al. (2006) constatou variações no crescimento das plantas e nos teores foliares de NPK. Todavia, nas condições de cultivo utilizadas as plantas sem adubação mostraram crescimento semelhante ao observado nas adu- badas. De modo geral, as plantas de alpínia apresentaram-se visualmente sadias, com crescimento vigoroso e sem sinais de deficiências nutricionais, inclusive no tratamento controle.

Assim como na adubação, raras são as informações sobre o manejo de água no cultivo de Zingiberaceae, especialmente de Alpinia. Lamas (2001) recomenda que a irrigação seja realizada periodicamente durante o cultivo de A. purpurata, enquanto Criley (1989) menciona que a exigência de água para a espécie é superior a $25 \mathrm{~mm}$ por semana durante períodos de estresse hídrico. Avaliando os efeitos do manejo de irrigação em $A$. zerumbet, Rezende et al. (2006) não observaram diferenças no crescimento e nos teores foliares de NPK quando as plantas foram submetidas aos idênticos dois limites de disponibilidade de água no solo usados no presente estudo (LDA1 - redução de $75 \%$ da capacidade total de retenção de água (CTA) - e LDA2 - redução de 50\% da CTA), no primeiro ano de cultivo.

O presente trabalho teve por objetivos avaliar a influência da adubação e da disponibilidade de água no solo sobre o teor e na composição química dos óleos essenciais em folhas de alpínia.

\section{MATERIAL E MÉTODOS}

O experimento foi instalado em agosto de 2002, na estação experimental do Centro de Ciências e Tecnologias Agropecuárias (CCTA) da Universidade Estadual do Norte Fluminense Darcy Ribeiro (UENF), localizada no município de Campos dos Goytacazes (RJ), situado a $21^{\circ} 45^{\circ}$ $15^{\prime \prime} \mathrm{S}$ e $41^{\circ} 19^{\prime} 28^{\prime \prime} \mathrm{W}$ e 13 metros de altitude (Miranda et al., 2005). A região, segundo Köppen, é classificada como tropical chuvosa, clima de bosque $(A m)$, variando a temperatura média mensal entre $21,4^{\circ} \mathrm{C}$, no mês mais frio, e $27,7^{\circ} \mathrm{C}$, no mês mais quente. A precipitação média anual é de $1.023 \mathrm{~mm}$, com chuvas concentradas nos meses de outubro a março (LEAG/UENF, 2002). Os dados meteorológicos, relativos ao período de condução do experimento, foram obtidos na estação meteorológica da Estação Experimental de Campos - PESAGRO, em Campos dos Goytacazes, RJ (Tabela 1).

O solo da área experimental, Neossolo Flúvico tb Eutrófico, apresentou as seguintes características químicas nas profundidades de 0-20 e 20-40 cm, respectivamente: $\mathrm{pH}$ (água 1:2,5) = 5,1 e 4,6; $\mathrm{P}=21$ e $12 \mathrm{mg} \mathrm{kg}^{-1} ; \mathrm{K}=$ 0,29 e $0,09 \mathrm{cmol}_{\mathrm{c}} \mathrm{kg}^{-1} ; \mathrm{Ca}=3,1$ e 2,4 $\mathrm{cmol}_{\mathrm{c}} \mathrm{kg}^{-1} ; \mathrm{Mg}=2,2 \mathrm{e}$ $1,6 \mathrm{cmol}_{\mathrm{c}} \mathrm{kg}^{-1} ; \mathrm{Al}=0,8$ e $1,7 \mathrm{cmol}_{\mathrm{c}} \mathrm{kg}^{-1} ; \mathrm{H}+\mathrm{Al}=9,1$ e 9,7 $\mathrm{cmol}_{\mathrm{c}} \mathrm{kg}^{-1} ; \mathrm{Na}=0,11$ e $0,14 \mathrm{cmol}_{\mathrm{c}} \mathrm{kg}^{-1}$; matéria orgânica $=$ 31,2 e 25,9 $\mathrm{g} \mathrm{kg}^{-1} ; \mathrm{SB}=5,7$ e 4,2 $\mathrm{cmol}_{\mathrm{c}} \mathrm{kg}^{-1} ; \mathrm{CTC}(\mathrm{T})=14,8$ e 13,9 $\mathrm{cmol} \mathrm{kg}_{\mathrm{c}} \mathrm{kg}^{-1}$ CTC (t) $=6,5$ e 5,9 $\mathrm{cmol} \mathrm{kg}_{\mathrm{c}} \mathrm{kg}^{-1} ; \mathrm{m}=12 \mathrm{e}$ $29 \%$; e V $=39$ e $30 \%$. As características físicas nas camadas de solo de 0-20 e 20-40 cm, respectivamente, são as 
seguintes: capacidade de campo $=46,5$ e 53,8\%; ponto de murcha $=29,4$ e 31,7\%; e densidade do solo $=1,11 \mathrm{~g} \mathrm{e} 1,12$ $\mathrm{g} \mathrm{cm}^{-3}$. Em relação à granulometria, na profundidade de $80-100 \mathrm{~cm}$ foram observadas as proporções de $5 \%$ de areia total, $38 \%$ de silte e $57 \%$ de argila. Antes do plantio o solo foi arado e gradeado e foi feita a incorporação de $1.500 \mathrm{~kg}$ ha ${ }^{-1}$ de calcário dolomítico.

As mudas constituíram-se de rizomas com torrão, retiradas de touceiras provenientes de um único clone cultivado na área experimental da Unidade de Apoio à Pesquisa/CCTA/UENF. Os rizomas foram padronizados com três pseudocaules seccionados em bisel a $20 \mathrm{~cm}$ da base. Os torrões tinham, aproximadamente, $20 \mathrm{~cm}$ de diâmetro por $20 \mathrm{~cm}$ de comprimento e pesavam cerca de $4 \mathrm{~kg}$. O experimento foi conduzido em blocos ao acaso, em esquema de parcelas subdivididas, com três repetições. As parcelas corresponderam a dois limites de umidade no solo para promover a reposição de água à cultura [limite de disponibilidade da água no solo 1 (LDA1) - redução de $75 \%$ da capacidade total de retenção de água (CTA) - e limite de disponibilidade da água no solo 2 (LDA2) - redução de $50 \%$ da CTA]. Cada tratamento (LDA) foi constituído por cinco linhas de 15 plantas $\left(300 \mathrm{~m}^{2}\right)$. As subparcelas foram representadas por cinco tipos de adubação (esterco bovino, cama-de-galinha, torta-de- filtro de cana-de-açúcar, adubo químico e sem adubação), contendo cinco linhas de três plantas $\left(60 \mathrm{~m}^{2}\right)$. O espaçamento usado foi de 2,0 x 2,0 m, sendo consideradas úteis as três plantas centrais de cada subparcela $\left(12 \mathrm{~m}^{2}\right)$.

A irrigação foi realizada por gotejamento, com vazão de aproximadamente $7,7 \mathrm{~L} \mathrm{~h}^{-1}$ por gotejador. Foram distri- buídos dois gotejadores por planta, espaçados a $50 \mathrm{~cm}$ entre si. Assim, a lâmina d'água aplicada por planta correspondeu a $4 \mathrm{~mm} \mathrm{~h}^{-1}$. A irrigação foi realizada em razão da capacidade total de água no solo (CTA), a qual é dada pela equação: $\mathrm{CTA}=\mathrm{DTA} * \mathrm{Z}$, em que DTA $=(\mathrm{CC}-\mathrm{PMP}) /$ $10 * \mathrm{D}_{\mathrm{a}}$, sendo DTA = disponibilidade de água no solo $\left(\mathrm{mm} \mathrm{cm}^{-1}\right) ; \mathrm{CC}=$ capacidade de campo do solo (\% peso); $\mathrm{PMP}=$ ponto de murcha permanente $(\%$ peso $) ; \mathrm{D}_{\mathrm{a}}=$ densidade aparente $\left(\mathrm{g} \mathrm{cm}^{-3}\right)$; e $\mathrm{Z}=$ profundidade da camada de solo $(20 \mathrm{~cm})$.

Verificado o pegamento das mudas, no terceiro mês após o plantio a irrigação foi diferenciada, sendo aplicados dois limites de umidade no solo, nos quais era feita a reposição de água à cultura. $\mathrm{O}$ balanço hídrico no solo foi estimado utilizando-se a equação: $\operatorname{Def}_{\mathrm{i}}=\operatorname{Def}_{\mathrm{i}-1}-(\mathrm{I}+$ $\mathrm{P})+\mathrm{ET}_{\mathrm{i}}$, em que Def $\mathrm{D}_{\mathrm{i}}$ déficit no final do período i $(\mathrm{mm})$; $\operatorname{Def}_{(\mathrm{i}-1)}=$ déficit no final do período i-1 (mm); I = irrigação $(\mathrm{mm}) ; \mathrm{P}=$ precipitação pluviométrica $(\mathrm{mm}) ;$ e $^{E_{i}}=$ evapotranspiração da cultura (mm). A evapotranspiração da cultura $\left(\mathrm{ET}_{\mathrm{i}}\right)$ é dada pela fórmula: $\mathrm{ET}_{\mathrm{i}}=\mathrm{K}_{\mathrm{c}} \mathrm{ET}_{0}$, sendo $\mathrm{ET}_{0}=$ evapotranspiração de referência e $\mathrm{K}_{\mathrm{c}}=$ coeficiente cultural, adimensional. Assumindo-se $\mathrm{K}_{\mathrm{c}}=1, \mathrm{ET}_{\mathrm{i}}=\mathrm{ET}_{0}$ a $\mathrm{ET}_{0}$ diária $\left(\mathrm{mm} \mathrm{d}^{-1}\right)$ foi calculada utilizando-se o método de Hargreaves - Samani (Pereira et al., 1997) - e estimada por $\mathrm{ET}_{0}=0,0023 \mathrm{Q}_{0}\left(\mathrm{~T}_{\text {máx. }}-\mathrm{T}_{\mathrm{mm}}\right)^{0,5}(\mathrm{~T}+17,8)$, em que $\mathrm{Q}_{0}$ é a radiação no topo da atmosfera $\left(\mathrm{mm} \mathrm{d}^{-1}\right) ; \mathrm{T}_{\text {máx. }}$ é a temperatura máxima; $\mathrm{T}_{\text {mín. }}$ é a temperatura mínima; e $\mathrm{T}$ é a temperatura média diária. $\mathrm{O}$ valor de $\mathrm{Q}_{0}$ foi estimado para a região Norte Fluminense (Sousa et al., 1996). O balanço hídrico nas parcelas, LDA1 e LDA2, é apresentado na Tabela 2 .

Tabela 1. Médias mensais de temperaturas máxima $\left(\mathrm{T}_{\text {máx }}\right.$ ), média $(\mathrm{T})$ e mínima $\left(\mathrm{T}_{\text {mín. }}\right)$, umidade relativa do ar (UR), radiação solar $(\mathrm{Rs})$, velocidade do vento $\left(\mathrm{U}_{2}\right)$, evapotranspiração $\left(\mathrm{ET}_{0}\right)$ e precipitação total $(\mathrm{PP})$ observadas na estação meteorológica da Estação Experimental de Campos - PESAGRO, em Campos dos Goytacazes (RJ)

\begin{tabular}{lcccccccc}
\hline Mês/ano & $\mathbf{T}_{\text {máx }}$ & $\mathbf{T}$ & $\mathbf{T}_{\text {min }}$ & $\mathbf{U R}(\mathbf{m m})$ & $\mathbf{R s}\left(\mathbf{w a t t s} \mathbf{~ m}^{-\mathbf{2}}\right)$ & $\mathbf{U}_{\mathbf{2}}\left(\mathbf{m ~ s}^{\mathbf{- 1}}\right)$ & $\mathbf{E T}_{\mathbf{0}}(\mathbf{m m})$ & $\mathbf{P P}(\mathbf{m m})$ \\
\hline Ago./02 & 28,5 & 20,3 & 17,8 & 76,0 & 190 & 2,2 & 3,92 & 19,80 \\
Set./02 & 25,1 & 20,6 & 17,0 & 77,7 & 171 & 2,4 & 3,41 & 146,50 \\
Out./02 & 30,3 & 24,0 & 19,7 & 74,3 & 258 & 2,8 & 5,50 & 27,30 \\
Nov./02 & 30,6 & 24,7 & 20,9 & 74,1 & 262 & 2,6 & 5,56 & 61,50 \\
Dez./02 & 31,1 & 25,5 & 22,0 & 78,7 & 242 & 2,0 & 5,03 & 114,80 \\
Jan./03 & 32,4 & 26,0 & 22,3 & 78,6 & 238 & 1,8 & 5,06 & 226,08 \\
Fev./03 & 33,7 & 26,9 & 21,8 & 71,9 & 296 & 2,0 & 6,15 & 15,50 \\
Mar./03 & 33,1 & 27,5 & 21,9 & 72,7 & 226 & 1,8 & 4,96 & 153,30 \\
Abr./03 & 30,6 & 24,4 & 20,3 & 75,7 & 191 & 1,5 & 3,80 & 82,10 \\
Mai./03 & 27,6 & 22,5 & 17,4 & 77,3 & 165 & 1,5 & 3,01 & 88,40 \\
Jun./03 & 28,9 & 21,7 & 16,9 & 77,6 & 164 & 1,4 & 2,98 & 0,40 \\
Jul./03 & 26,8 & 20,3 & 15,5 & 79,4 & 156 & 1,7 & 2,99 & 35,20 \\
Ago./03 & 25,4 & 19,9 & 15,7 & 77,1 & 181 & 1,9 & 3,30 & 50,30 \\
Set./03 & 27,2 & 22,8 & 18,5 & $*$ & $*$ & $*$ & $*$ & 39,30 \\
Out./03 & 28,7 & 24,0 & 19,4 & $*$ & $*$ & $*$ & $*$ & 66,90 \\
Nov./03 & 30,7 & 26,0 & 21,4 & $*$ & $*$ & $*$ & $*$ & 83,90 \\
\hline
\end{tabular}

* Médias mensais não registradas de set./03 a nov./03 na estação meteorológica da Estação Experimental de Campos - PESAGRO, em Campos dos Goytacazes. 
As adubações de cobertura foram realizadas em três épocas: no plantio, aos seis e aos nove meses após o plantio. Antes de cada adubação, amostras dos adubos orgânicos foram submetidas à secagem em estufa com ventilação forçada a $70^{\circ} \mathrm{C}$ por 48 h e à trituração em moinho Wiley com peneira de 20 mesh. Em seguida, o material foi submetido à digestão sulfúrica (Malavolta et al., 1997). O nitrogênio orgânico ( $\mathrm{N}$ org) foi determinado pelo método de Nessler (Jackson, 1965). A determinação do fósforo (P) foi realizada pela redução do complexo fosfo-molíbdico pela vitamina $\mathrm{C}$ (Braga \& Defelipo, 1974). Os teores de N orgânico e P foram determinados por colorimetria de absorção atômica. A leitura de teores de potássio $(\mathrm{K})$ foi realizada em fotômetro de chama. Na determinação de nitrato $\left(\mathrm{NO}_{3}^{-}\right)$, utilizou-se o método colorimétrico do ácido salicílico (Cataldo et al., 1975), após submeter as amostras ao banho-maria, a $45^{\circ} \mathrm{C}$, por uma hora (Malavolta et al., 1997).

Diferentes adubos, orgânicos e minerais, foram empregados nas adubações das subparcelas do experimento. Na adubação orgânica utilizaram-se esterco bovino curtido, cama-de-galinha poedeira curtida e torta de filtro de usina de cana-de-açúcar, prontamente disponíveis na região. A cama-de-galinha poedeira, além de fezes, penas e ração, continha resíduos de madeira (maravalha).

Os teores de nitrogênio total ( $\mathrm{N}$ total), fósforo $(\mathrm{P})$ e potássio $(\mathrm{K})$ das amostras dos adubos orgânicos foram usados nos cálculos de adubação, de modo a fornecer 75 $\mathrm{kg} \mathrm{ha}^{-1}$ de N, $75 \mathrm{~kg} \mathrm{ha}^{-1}$ de $\mathrm{P}_{2} \mathrm{O}_{5}\left(32,75 \mathrm{~kg} \mathrm{ha}^{-1}\right.$ de P) e $37,5 \mathrm{~kg}$ ha $^{-1}$ de $\mathrm{K}_{2} \mathrm{O}\left(31,12 \mathrm{~kg} \mathrm{ha}^{-1}\right.$ de K), em cada adubação (Tabela 3). $\mathrm{O}$ teor de $\mathrm{P}$ foi usado para o cálculo em virtude da variação dos teores dos nutrientes nos adubos, e quando houve necessidade estes eram enriquecidos com fosfato de Araxá. Para a adubação mineral foram utilizados os fertilizantes: ureia (46\% de $\mathrm{N}$ solúvel em água), superfosfato simples ( $16 \%$ de $\mathrm{P}_{2} \mathrm{O}_{5}$ solúvel em água) e cloreto de potássio (60\% $\mathrm{K}_{2} \mathrm{O}$ solúvel em água).
Aos 12 meses após o plantio, folhas verdes de duas hastes vegetativas foram coletadas, entre 9 e 12 h, em cada planta da subparcela útil, acondicionadas em sacos de papel e encaminhadas para o laboratório, onde foram limpas, com papel-toalha seco, para remoção de poeira e fuligem. Em seguida, foram submetidas à secagem em estufa com circulação de ar à temperatura de $45^{\circ} \mathrm{C}$, onde permaneceram por oito dias. Nesse ponto, as folhas apresentavam-se bastante quebradiças ao serem manuseadas. As amostras secas foram trituradas manualmente e acondicionadas em sacos de papel pardo e mantidas em local seco até a extração do óleo essencial.

O método de extração do óleo essencial foi o de destilação a vapor (hidrodestilação), em extrator tipo Clevenger. O tempo de extração foi de aproximadamente $60 \mathrm{~min}$, contados após o escorrimento da primeira gota da condensação no tubo coletor graduado (bureta). As amostras de óleo essencial foram armazenadas em recipientes do tipo Eppendorf e mantidas em freezer até a determinação dos constituintes químicos delas. Nessa fase, determinou-se a concentração de óleo essencial (\%) com base no peso do óleo essencial (g) extraído em $100 \mathrm{~g}$ de folhas secas.

A qualidade do óleo essencial foi analisada por meio dos principais componentes químicos, e seus respectivos teores determinados pelo método de cromatografia gasosa, no Laboratório de Química do Centro de Ciências Tecnológicas (CCT), da UENF. Para tanto, utilizou-se o cromatógrafo a gás acoplado ao espectrômetro de massa e o cromatógrafo a gás com detector de íons por chama.

No cromatógrafo a gás acoplado ao espectrômetro de massa (CG-EM) foi utilizada a coluna DB1, cujo comprimento e diâmetro são $30 \mathrm{~m}$ e $0,25 \mathrm{~mm}$, respectivamente. A temperatura inicial da coluna foi de $50^{\circ} \mathrm{C}$, atingindo $230^{\circ} \mathrm{C}$ em uma razão de $15^{\circ} \mathrm{C}$ por minuto e permanecendo por sete minutos a $230^{\circ} \mathrm{C}$. A temperatura do injetor foi de $200^{\circ} \mathrm{C}$ e a da interface entre CG-EM, mantida em $230^{\circ} \mathrm{C}$. O fluxo foi de

Tabela 2. Balanço hídrico nas parcelas LDA1 e LDA2, em razão da precipitação (PP), lâmina de água aplicada (LA), frequência (Freq.) e turno de rega médio (TR)

\begin{tabular}{|c|c|c|c|c|c|c|c|}
\hline \multirow{2}{*}{ Mês } & \multirow{2}{*}{$\mathbf{P P}(\mathbf{m m})$} & \multicolumn{3}{|c|}{ LDA1 } & \multicolumn{3}{|c|}{ LDA2 } \\
\hline & & $\mathbf{L A}(\mathbf{m m})$ & Freq. & TR (dias) & $\mathbf{L A}(\mathbf{m m})$ & Freq. & TR (dias) \\
\hline Nov./02 & 61,5 & 64 & 1 & 30 & 44 & 2 & 15 \\
\hline Dez./02 & 114,8 & 64 & 1 & 30 & & & \\
\hline Jan./03 & 226,08 & & & & 44 & 1 & 30 \\
\hline Fev./03 & 15,5 & 64 & 3 & 10 & 44 & 3 & 10 \\
\hline Mar./03 & 153,3 & 64 & 1 & 30 & 44 & 2 & 15 \\
\hline Abr./03 & 82,1 & & & & & & \\
\hline Maio/03 & 88,4 & & & & & & \\
\hline Jun./03 & 0,4 & 64 & 1 & 30 & 44 & 1 & 30 \\
\hline Jul./03 & 35,2 & 64 & 1 & 30 & 44 & 1 & 30 \\
\hline Ago./03 & 50,3 & & & & 44 & 2 & 15 \\
\hline \multicolumn{2}{|c|}{ Total de água aplicada (mm) } & 512 & & & 528 & & \\
\hline
\end{tabular}

Parcelas não irrigadas no mês correspondente. 
Tabela 3. Teores de nitrogênio total ( $\mathrm{N}$ total), fósforo $(\mathrm{P})$ e potássio (K) em adubos orgânicos, em três épocas de aplicação

\begin{tabular}{lcccc}
\hline \multirow{2}{*}{ Época de adubação } & \multirow{2}{*}{ Adubo } & $\mathbf{N ~ t o t a l ~}^{\mathbf{1 /}}$ & $\mathbf{P}$ & $\mathbf{K}$ \\
\cline { 3 - 5 } & & \multicolumn{3}{c}{$\mathbf{~ g ~ k g}^{-\mathbf{1}}$} \\
\hline \multirow{3}{*}{ Plantio } & EB & 27,36 & 6,13 & 27,80 \\
& CG & 29,12 & 18,84 & 11,40 \\
& TF & 20,65 & 15,58 & 1,19 \\
\hline \multirow{3}{*}{6 meses } & EB & 39,06 & 7,82 & 21,90 \\
& CG & 69,76 & 19,29 & 11,35 \\
& TF & 32,26 & 17,51 & 1,50 \\
\hline \multirow{3}{*}{9 meses } & EB & 27,25 & 4,99 & 13,40 \\
& CG & 23,75 & 8,45 & 10,70 \\
& TF & 19,45 & 17,77 & 2,25 \\
\hline
\end{tabular}

Adubo: $\mathrm{EB}=$ esterco bovino; $\mathrm{CG}=$ cama-de-galinha; $\mathrm{e} \mathrm{TF}=$ torta de filtro.

${ }^{1 /} \mathrm{N}$ total $=\mathrm{N}$ org $+\mathrm{NO}_{3}{ }^{-}$.

$1,5 \mathrm{~mL} \mathrm{~min}^{-1}$. A pressão inicial foi $87,5 \mathrm{kPa}$, atingindo numa razão de $7 \mathrm{kPa} \min ^{-1} 171 \mathrm{kPa}$, na qual permaneceu por sete minutos. O split ratio do aparelho foi de 1:20.

No cromatógrafo a gás com detector de íons por chama a coluna utilizada também foi a DB1, com as mesmas dimensões do CG/EM. A temperatura inicial da coluna foi $50{ }^{\circ} \mathrm{C}$, atingindo $230{ }^{\circ} \mathrm{C}$ em uma razão de $15^{\circ} \mathrm{C}$ por minuto e permanecendo por sete minutos a $230^{\circ} \mathrm{C}$. A temperatura do injetor foi de $200^{\circ} \mathrm{C}$. O fluxo foi de $1,5 \mathrm{~mL} \mathrm{~min}^{-1} \mathrm{e}$ o fluxo total, $33 \mathrm{~mL} \mathrm{~min}^{-1}$. A pressão inicial foi $61 \mathrm{kPa}$ numa razão de $4 \mathrm{kPa} \min ^{-1}$, chegando a $109 \mathrm{kPa}$, permanecendo nesta pressão por sete minutos. A velocidade linear foi de 41,7147 x $10 \mathrm{~mm} \mathrm{seg}^{-1}$. O split ratio do aparelho foi de 1:20.

Os dados observados foram submetidos à análise de variância, utilizando-se o procedimento ANOVA. As comparações de médias foram efetuadas utilizando-se o teste de Tukey a 5\% de significância.

\section{RESULTADOS E DISCUSSÃO}

As análises estatísticas não apontaram diferenças entre os tratamentos de irrigação, tampouco entre os adubos utilizados na produção do óleo essencial e na maioria de seus componentes voláteis aos 12 meses após o plantio. Obteve-se um teor de aproximadamente $0,74 \%$ de óleo essencial em folhas secas de $A$. zerumbet. Esse resultado está de acordo com aqueles obtidos por Rezende et al. (2003), que verificaram variação nos teores do mesmo óleo entre 0,69 e 0,77\%. Em outro estudo, De Pooter et al. (1995), avaliando o teor de óleo essencial na matéria fresca de diferentes órgãos da planta de A. zerumbet, obtiveram baixos teores extraídos por hidrodestilação em folhas, rizomas e hastes, que foram, respectivamente, de $0,12,0,10$ e $0,05 \%$. Portanto, bem mais baixos que o observado na presente pesquisa. Entretanto, os autores não forneceram detalhes fitotécnicos sobre o cultivo, como adubação e manejo de irrigação.
Dentre os monoterpenos identificados nas amostras de óleo essencial de A. zerumbet, o sabineno, 1,8-cineol e 1terpinen-4-ol foram os encontrados em maior concentração (Tabela 4). Esses resultados estão de acordo com aqueles obtidos por Zoghbi et al. (1999) e Rezende et al. (2003). Altos teores de 1,8-cineol (23,1\%), 1-terpinen-4-ol $(22,7 \%)$ e sabineno (14,5\%) estão presentes em óleo essencial dessa espécie (Zoghbi et al.,1999). Rezende et al. (2003) também verificaram altos teores de 1-terpinen-4-ol (21,53 a 23,80\%) e 1,8-cineol (20,43 a 22,67\%). Entretanto, para o sabineno os mesmos autores verificaram teores bem mais baixos $(5,85$ a 7,29\%), embora ainda sendo constituinte majoritário.

Em outros estudos, os principais constituintes do óleo essencial de $A$. speciosa foram $\alpha$-pineno, $\beta$-pineno, 1,8 cineol e $\rho$-cimeno, tendo os dois últimos compostos apresentado altos teores, 22,4 e 20,8\%, respectivamente (Gottlieb et al., 1981). Nos óleos essenciais extraídos de folhas secas ao ar, provenientes de duas espécies de Zingiberaceae cultivadas em Manaus, Belém e no Amazonas, Luz et al. (1985) observaram para A. speciosa que, além do cineol $(14,87 \%)$, outros constituintes em maior concentração, como 4-terpineol (20,40\%), $\rho$-cimeno (9,38\%), $\gamma$-terpineno $(9,48 \%)$ e sabineno $(5,97)$, foram identificados. Entretanto, no óleo essencial de Rengalmia floribunda o $\beta$-pineno foi considerado o principal componente volátil.

Quanto ao fator disponibilidade de água no solo, apenas o teor do 1,8-cineol apresentou diferença, sendo maior na parcela LDA2 (Tabela 4). O cineol ou eucaliptol representa um dos principais compostos do óleo essencial de Eucalyptus globulus Labill de grande interesse para as indústrias de perfumaria, alimentícia e farmacêutica (Almeida et al., 2005). Ao contrário deste estudo, outras pesquisas têm demonstrado que quando determinada espécie é submetida ao estresse hídrico a concentração de seus princípios ativos tende a aumentar. Esse fato foi verificado por Dovrat e Goldschimidt (1978) em plantas de Catharanthus roseus, que em regime de solo seco apresentaram maior teor de ajmalicina nas raízes do que em condições de solo úmido. Por outro lado, em plantas de Nicotiana glauca o teor de alcaloides diminuiu com o turno de rega a cada 10 dias e aumentou com intervalos maiores entre irrigações de 30 ou 40 dias. No entanto, averiguou-se, no mesmo trabalho, que maior percentagem de rutina ocorreu em intervalos mais curtos de irrigação (Saleh et al., 1978).

O teor do $\beta$-pineno foi o único que variou de acordo com os adubos usados (Tabela 4), sendo menor em plantas adubadas com esterco bovino, enquanto nos demais tratamentos os teores de $\beta$-pineno foram semelhantes aos da testemunha, sem adubação, sugerindo que a adubação tem pouca influência no rendimento e na qualidade do óleo essencial de alpínia. Verificou-se que, no primeiro ano de produção, foram extraídos aproximadamente 7,40 
Tabela 4. Teor de óleo essencial e seus principais constituintes químicos em folhas de alpínia, em razão da disponibilidade de água no solo (LDA) e da adubação (adubo) aos 12 meses após o plantio

\begin{tabular}{|c|c|c|c|c|c|c|c|c|}
\hline \multirow{2}{*}{ LDA } & \multirow{2}{*}{ Óleo (\%) } & \multicolumn{7}{|c|}{ Principais constituintes químicos $(\%)$} \\
\hline & & $\alpha$-thujeno & $\alpha$-pineno & sabineno & $\beta$-pineno & $\beta$-mirceno & 1,8-cineol & 1-terpinen-4-ol \\
\hline 1 & $0,74 \mathrm{a}$ & $6,09 \mathrm{a}$ & $2,73 \mathrm{a}$ & $17,02 \mathrm{a}$ & $4,66 \mathrm{a}$ & $1,82 \mathrm{a}$ & $19,31 \mathrm{~b}$ & $14,02 \mathrm{a}$ \\
\hline 2 & $0,73 \mathrm{a}$ & $6,13 \mathrm{a}$ & $2,66 \mathrm{a}$ & 17,02 a & $4,62 \mathrm{a}$ & $1,70 \mathrm{a}$ & $19,51 \mathrm{a}$ & 14,62 a \\
\hline \multicolumn{9}{|l|}{ Adubo } \\
\hline $\mathrm{CT}$ & $0,75 \mathrm{a}$ & $6,02 \mathrm{a}$ & $2,82 \mathrm{a}$ & $16,28 \mathrm{a}$ & $4,73 \mathrm{ab}$ & $1,93 \mathrm{a}$ & $19,25 \mathrm{a}$ & $14,16 \mathrm{a}$ \\
\hline CG & $0,75 \mathrm{a}$ & $6,18 \mathrm{a}$ & $2,51 \mathrm{a}$ & $16,23 \mathrm{a}$ & $4,80 \mathrm{a}$ & $1,91 \mathrm{a}$ & 19,24 a & $13,82 \mathrm{a}$ \\
\hline $\mathrm{TF}$ & $0,71 \mathrm{a}$ & $6,20 \mathrm{a}$ & $2,51 \mathrm{a}$ & $16,93 \mathrm{a}$ & $4,46 \mathrm{ab}$ & $1,47 \mathrm{a}$ & $19,60 \mathrm{a}$ & $14,74 \mathrm{a}$ \\
\hline AQ & $0,72 \mathrm{a}$ & $6,07 \mathrm{a}$ & $2,81 \mathrm{a}$ & $17,06 \mathrm{a}$ & $4,94 \mathrm{a}$ & $1,86 \mathrm{a}$ & $19,41 \mathrm{a}$ & $14,17 \mathrm{a}$ \\
\hline $\mathrm{EB}$ & $0,75 \mathrm{a}$ & $6,07 \mathrm{a}$ & $2,52 \mathrm{a}$ & 16,96 a & $4,27 \quad b$ & $1,62 \mathrm{a}$ & $19,56 \mathrm{a}$ & $14,72 \mathrm{a}$ \\
\hline Média & 0,74 & 6,11 & 2,69 & 16,69 & 4,64 & 1,76 & 19,41 & 14,32 \\
\hline$\overline{\text { C.V.(\%) }}$ & 5,39 & 6,92 & 8,81 & 9,87 & 6,28 & 17,17 & 1,89 & 6,45 \\
\hline
\end{tabular}

Adubo: $\mathrm{CT}=$ controle, $\mathrm{CG}=$ cama-de-galinha, $\mathrm{TF}=$ torta-de-filtro, $\mathrm{AQ}=$ adubo químico e $\mathrm{EB}=$ esterco bovino.

Médias seguidas de mesmas letras na coluna, em razão da disponibilidade de água no solo ou do adubo, não diferem entre si pelo teste de Tukey $(\mathrm{P}>0,05)$

$\mathrm{g}$ de óleo em $1.000 \mathrm{~g}$ de folhas secas, independentemente do manejo de água no solo e dos adubos utilizados.

Rezende et al. (2003), trabalhando com doses crescentes de nitrogênio $(0,10,20$ e $30 \mathrm{~g}$ de N/cova), não observaram diferenças sobre a composição do óleo de alpínia. Em Datura stramonium, as doses de $\mathrm{N}$ relativamente altas reduziram a concentração de alcaloides em plantas jovens; todavia, esse efeito foi revertido em plantas adultas (Demeyer e Dejaegere, 1997).

Os efeitos dos adubos orgânicos no rendimento e na qualidade dos óleos essenciais têm sido pesquisado (Singh et al., 1988; Scheffer \& Ronzelli Junior, 1993; Silva et al., 2002). Porém, na recomendação da adubação orgânica para plantas medicinais, além do rendimento da biomassa deve-se também levar em conta o teor de seus princípios ativos (Ming, 1998). Em Lippia alba, a adubação com esterco bovino causou aumento da produção de biomassa; no entanto, ocasionou redução do teor de óleo à medida que aumentaram as doses do esterco. Essa correlação inversa entre o teor de óleo e biomassa, segundo Ming (1994), pode ser atribuída ao fato de que o óleo, produto resultante do metabolismo secundário, desempenha função de defesa na planta e de que em condições menos favoráveis pode ocorrer maior síntese de óleo, o que não foi constatado pelo autor.

A adubação orgânica com $1 \mathrm{~kg} \mathrm{~m}^{-2}$ de esterco de ave trouxe aumento do teor de óleo essencial de gengibre (Zingiber officinale); porém, não houve efeito sobre a composição do óleo essencial (Silva et al., 2002). Em plantas de alfavaca-cravo (Ocimum gratissimum), a adubação com diferentes doses de esterco curtido de poedeira $(0,4$, 8 e $12 \mathrm{~kg} \mathrm{~m}^{-2}$ ) não mostrou diferenças sobre o rendimento do óleo e teor eugenol, mas na ausência do adubo orgânico constatou-se menor produção de folhas por planta (Chaves et al., 2002).
Trabalhos relacionados ao manejo da cultura com ênfase no crescimento e florescimento de A. zerumbet têm obtido poucos resultados. Com a aplicação de doses crescentes de nitrogênio $(0,10,20$ e $30 \mathrm{~g}$ de N/cova) adicionadas de $30 \mathrm{~g} /$ cova de $\mathrm{P}_{2} \mathrm{O}_{5}(13,10 \mathrm{~g} /$ cova de $\mathrm{P})$ e $15 \mathrm{~g} /$ cova de $\mathrm{K}_{2} \mathrm{O}$ (12,45 g/cova de $\mathrm{K}$ ), Jasmim et al. (2001) observaram apenas aumento linear na altura da haste maior, não sendo constatado efeito da adubação nitrogenada para número de perfilhos e de folhas por planta aos seis meses após o plantio.

Rezende et al. (2006) comprovaram que tanto o manejo hídrico como os adubos orgânicos e químicos tiveram pouco efeito sobre o crescimento e os teores foliares de NPK em alpínia e que praticamente não houve resposta desses fatores sobre as características de florescimento avaliadas no primeiro ano de cultivo. Esses resultados podem ser atribuídos à rusticidade da espécie, que nas condições de cultivo utilizadas apresentou crescimento bastante homogêneo e vigoroso, sem sintomas visuais de deficiência nutricional, mesmo no tratamento controle, não adubado.

\section{CONCLUSÕES}

Os limites de reposição de água no solo não influenciam a produção de óleo essencial de alpínia nem na maioria de seus constituintes químicos. A adubação também tem pouca influência no rendimento e na qualidade desse óleo essencial. No primeiro ano de produção, são extraídos, aproximadamente, 7,40 g de óleo em $1.000 \mathrm{~g}$ de folhas secas, independentemente do manejo de irrigação e dos adubos utilizados. Os principais constituintes químicos presentes em óleos essenciais de alpínia são: $\alpha$-thujeno $(6,11 \%), \alpha$-pineno $(2,69 \%)$, sabineno $(16,69 \%), \beta$-pineno (4,64\%), $\beta$-mirceno $(1,76 \%), 1,8$-cineol $(19,41 \%)$ e 1 terpinen-4-ol $(14,32 \%)$. 


\section{AGRADECIMENTOS}

À UENF, pela concessão da bolsa de estudos, e à FAPERJ, pelo financiamento do projeto. À FENORTE/ TECNORTE, pela concessão de bolsa de Apoio Técnico.

\section{REFERÊNCIAS BIBLIOGRÁFICAS}

Alencar JW, Silva MG de V, Machado MIL, Craveiro AA, Matos FJ de A \& Magalhães R de A (1997) Use of ${ }^{13} \mathrm{C}-\mathrm{NMR}$ as complementar identification tool in essential oil analysis. Spectroscopy, 13:265-273.

Alice CB, Siqueira NCS, Mentz LA, Brasil e Silva GA de A \& José KFD (1995) Plantas medicinais de uso popular: atlas farmacognóstico. Canoas, Editora da ULBRA. 205p.

Almeida LP, Ferri PH, Paula JR \& Santiago MF (2005) Biotransformaçâo do 1,8-cineol por bactérias livres e Imobilizadas. Revista Eletrônica de Farmácia, 2:1-5.

Bezerra MAC, Leal-Cardoso JH, Coelho-de-Souza A, Criddle DN \& Fonteles MC (2000) Myorelaxant and antispasmodic effects f essential oil of Alpinia speciosa on rat ileum. Phytotherapy Research, 14:549-551.

Braga JM \& Defelipo BV (1974) Determinação espectrofotométrica de fósforo em extratos de solos e material vegetal. Revista Ceres, 21:73-85.

Cataldo DA, Haroon M, Schrader LE \& Young UL (1975) Rapid colorimetric determination of nitrate in plant tissue by nitration of salicylic acid. Communications In Soil Science And Plant Analysis, 6:71-80.

Chaves EC de O (2001) Efeito do extrato hidroalcoólico de Alpinia zerumbet na estrutura e remodelagem do miocárdio na hipertensão causada por bloqueio da síntese do óxido nítrico em ratos. Dissertação de Mestrado. Universidade Estadual do Rio de Janeiro, Rio de Janeiro. 44p.

Chaves FCM, Ming LC, Carvalho EAV, Fernandes DM, Marques MOM \& Meireles MAA (2002) Produção de biomassa, rendimento de óleo essencial e teor de eugenol em alfavaca-cravo, em função da adubação orgânica e sazonalidade. Horticultura Brasileira, 20:310.

Côrrea MP (1974) Dicionário das plantas úteis do Brasil e das exóticas cultivadas. Rio de Janeiro, IBDF, 5:687p.

Criley RA (1989) Development of heliconia and Alpinia in Hawai: cultivar selection and cultura. Acta Horticulturae, 246: 247-258.

De Pooter HL, Aboutabl EA \& El-Shabrawy AO (1995) Chemica composition and antimicrobial activity of essential oil of leaf, stem and rhizome of Alpinia speciosa (J. C. Wendl.) K. Schum. Grow in Egypt. Flavour and Fragrance Journal, 1:63-67.

Dovrat A \& Goldschmidt J (1978) Cultivation aspects of Catharanthus roseus for roots. Acta Horticulturae, 73:263-268

Demeyer K \& Dejaegere R (1997) Nitrogen and alkaloid accumulation and partitioning in Datura stramonium L. Journal of Herbs, Spices and Medicinal Plants, 5:15-23.

Gottlieb OR, Koketsu M, Magalhães MT, Maia JGS, Mendes PH Rocha AL da, Silva ML da \& Wilberg VC (1981) Óeos essenciais da Amazônia VII. Acta Amazônica, 11:143-148.

Jackson M.L. (1965) Soil chemical analysis, 5 ed. Englewood Cliffs, Prentice-Hall. 498p.

Jasmim J, Prado MA, Silva JCC \& Carvalho CM (2001) A adubação nitrogenada no crescimento vegetativo de Alpinia zerumbet (Pers.) Burtt e Smith. In: $13^{\circ}$ Congresso Brasileiro de Floricultura e Plantas Ornamentais, São Paulo. Anais, SBFPO. v.1, p.109.
Lahlou S, Galindo CA, Leal-Cardoso JH, Fonteles MC \& Duarte GP (2002) Cardiovascular effects of the essential oil of Alpinia zerumbet leaves and its main constituent, terpinen-4-ol, in rats: role of the autonomic nervous system. Planta Medica, 68:10971102 .

Lahlou S, Interaminense LFL, Leal-Cardoso JH \& Duarte GP (2003) Antihypertendive effects of the essential oil of Alpinia zerumbet and ists main constituent, terpinen-4-ol, in DOCAsalt hypertensive conscious rats. Fundamental and Clinical Pharmacology, 17:323-330.

Lamas A da M (2001) Floricultura tropical: técnicas de cultivo. Recife, SEBRAE/PE. 88p.

Larsen K, Ibrahim H, Khaw SH \& Saw LG (1999) Gingers of Peninsular Malaysia and Singapore. Kota kinabalu, Natural History Publications/Sabah. 135p.

LEAG/UENF - Laboratório de Engenharia Agrícola da Universidade Estadual do Norte Fluminense (2002) Dados agrometerorológicos de Campos dos Goytacazes- RJ. Convênio PESAGRORIO/UENF. Setor de irrigação e agrometerologia.

Lorenzi H \& Souza HM de (2001) Plantas Ornamentais no Brasil: arbustivas, herbáceas e trepadeiras. 3 ed. Nova Odessa, Plantarum. $1088 \mathrm{p}$.

Lorenzi H \& Matos FJA (2002) Plantas medicinais no Brasil: nativas e exóticas cultivadas. Nova Odessa, Instituto Plantarum. 512 p.

Luz AIR, Zoghbi MGB, Ramos LS, Maia JGS \& Silva ML (1985) Essential oils of some Amazonian Zingiberaceae, 3. Genera Alpinia and Rengalmia. Journal Natural Products, 47:907-908.

Malavolta E, Vitti GC \& Oliveira AS (1997) Avaliação do Estado Nutricional das Plantas. Princípios e aplicações. Piracicaba, Associação Brasileira para Pesquisa da Potassa e do Fosfato (POTAFOS). 319p.

Matos FJA (2002) Farmácias vivas: sistema de utilização de plantas medicinais projetado para pequenas comunidades. 4. ed. Fortaleza, UFC. 267p.

Ming LC (1994) Influência da adubação orgânica na produção de biomassa e teor de óleos essenciais de Lippia alba. Horticultura Brasileira, 12:49-51.

Ming LC (1998) Adubação orgânica no cultivo de Lippia Alba (Mill) N. E. Br. - Verbenaceae. In: Plantas medicinais, aromáticas e condimentares: avanços na pesquisa agronômica. Botucatu, UNESP, 1:165-191.

Miranda EE de, Gomes EG \& Guimarães M (2005) Mapeamento e estimativa da área urbanizada do Brasil com base em imagens orbitais e modelos estatísticos. Campinas, Embrapa Monitoramento por Satélite. Disponível em: <http://www.urbanizacao.cnpm.embrapa.br>. Acessado em: 7 maio de 2008.

Panizza S (1997) Plantas que curam: cheiro de mato. 24 ed. São Paulo, IBRASA. 279 p.

Pereira AR, Villa NA \& Sediyama GC (1997) Evapotranspiração. Piracicaba, ESALQ. 183p.

Rezende ME de, Jasmim J, Prado MA do, Caprini GP \& Shripsema J (2003) Efeito da adubação nitrogenada no rendimento e qualidade do óleo essencial de colônia (Alpinia zerumbet). In: $2^{\circ}$ Simpósio Brasileiro de Óleos Essenciais, Capinas. Anais, IAC. p.46.

Rezende ME de, Jasmim JM, Sousa EF de, Thiébaut JTL, Carvalho, AJC de \& Pinheiro MM (2006) Crescimento, florescimento e teores foliares de NPK em alpínia: influência da adubação e irrigação. Revista Ceres, 53:635-644.

Saleh MM, Makarem M \& El-Gamasy AM (1978) Effect of irrigation on the growth, alkaloids and rutin of Nicotiana glauca. Acta Horticulturae, 73:199-204. 
Scheffer MC \& Ronzelli Junior P (1993) Influence of organic fertilization on the biomass, yield and composition of the essential oil of Achillea millefolium L. Acta Horticulturae, 331:109-114.

Silva MAS da, Marques MOM \& Ming LC (2002) Influência da adubação orgânica no teor e na composição do óleo essencial de gengibre. Horticultura Brasileira, 20:308.

Singh K, Singh V \& Ram P (1988) Effect of farmyard manure and fertilizers on herb, oil and sucker yield of Mentha arvensis L. Indian Journal Agronomy, 33:287-289.
Sousa EF de, Bernardo S \& Carvalho J de A (1996) Um método prático para a estimativa de $\mathrm{ET}_{0}$ com dados de temperatura máxima e mínima para as "áreas de baixada e de tabuleiros" da região Norte-Fluminense (equação de Hargreaves). Campos dos Goytacazes, UENF. 15p. (Boletim $\mathrm{n}^{\mathrm{o}} 2$ ).

Zoghbi M das GB, Andrade EHA \& Maia JGS (1999) Volatile constituents from leaves and flowers of Alpinia speciosa $\mathrm{K}$. schum. And A. purpurata (Viell.) Schum. Flavour and Fragrance Journal, 1:411-414. 